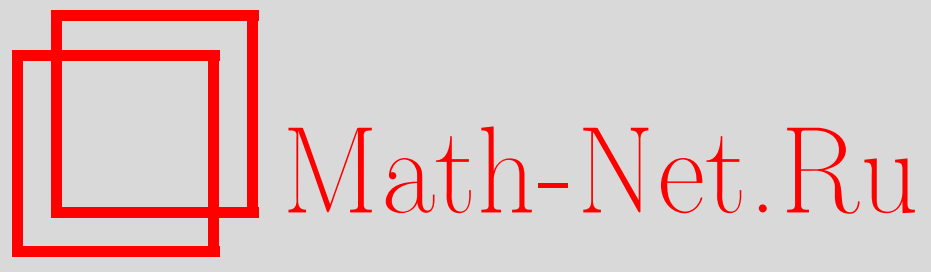

И. В. Лосев, Классификация слабо коммутативных комплексных однородных пространств, УМН, 2007, том 62, выпуск 2, 181-182

DOI: https://doi.org/10.4213/rm6426

Использование Общероссийского математического портала Math-Net.Ru подразумевает, что вы прочитали и согласны с пользовательским соглашением http://www . mathnet.ru/rus/agreement

Параметры загрузки:

IP : 54.164 .48 .24

26 апреля 2023 г., 12:35:02

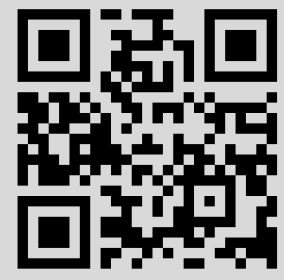




\section{Классификация слабо коммутативных комплексных однородных пространств}

\section{И. В. Лосев}

Пусть $G$ - комплексная связная алгебраическая группа, а $H$ - ее алгебраическая подгруппа. Отметим, что многообразие $T^{*}(G / H)$ наделяется естественной симплектической формой, что задает скобку Пуассона на поле реальных функций $\mathbb{C}\left(T^{*}(G / H)\right)$.

ОПределЕниЕ 1 . Однородное пространство $G / H$ называется слабо коммутативным, если поле рациональных инвариантов $\mathbb{C}\left(T^{*}(G / H)\right)^{G}$ коммутативно относительно скобки Пуассона, индуцированной с $\mathbb{C}\left(T^{*}(G / H)\right)$.

Цель этой работы - классификация слабо коммутативных однородных пространств с редуктивным стабилизатором $H$ при некоторых технических ограничениях.

Ниже алгебра Ли алгебраической группы, обозначаемой прописной латинской буквой, обозначается строчной готической буквой.

Обсудим некоторые результаты, полученные ранее в том же направлении. Известна классификация в случае, когда и $G$, и $H$ редуктивны, ее обзор дан в [1]. В работах [1]-[4] рассматривался случай вещественной группы Ли $G$ и компактной подгруппы $H$ (здесь в определении слабой коммутативности рассматривают алгебру всех инвариантных гладких функций на $T^{*}(G / H)$, полиномиальных на слоях расслоения $\left.T^{*}(G / H) \rightarrow G / H\right)$. В [2] были классифицированы все слабо коммутативные пространства вида $(N \lambda H) / H$, где $H$ - компактная группа Ли, а $N$ - односвязная нильпотентная группа Ли, на которой $H$ действует автоморфизмами, причем представление $H$ в $\mathfrak{n} /[\mathfrak{n}, \mathfrak{n}]$ неприводимо. Классификация в общем случае была проведена в [3], ее результаты частично представлены также в [4]. В работе [5] рассмотрен случай комплексного однородного пространства $(N \lambda H) / H$ с редуктивным стабилизатором $H$. Там получена классификация при технических ограничениях, аналогичных введенным в [2].

Приведем теперь основной классификационный результат. Мы рассматриваем однородные пространства вида $(N \lambda L) / H$, где $L$ - связная редуктивная группа, $H$ - ее связная редуктивная подгруппа, $N$ - некоммутативная унипотентная группа, на которой $L$ действует автоморфизмами, причем $L$-модуль $\mathfrak{n} /[\mathfrak{n}, \mathfrak{n}]$ неприводим. Отметим, что слабая коммутативность пространства $G / H$ зависит лишь от пары $(\mathfrak{g}, \mathfrak{h})$. Мы говорим, что пара $(\mathfrak{g}, \mathfrak{h})$ (соответственно тройка $(\mathfrak{n}, \mathfrak{l}, \mathfrak{h}))$ слабо коммутативна, если однородное пространство $G / H$ (соответственно $(N \lambda L) / H$ ) таково.

Мы говорим, что тройка $(\mathfrak{n}, \mathfrak{l}, \mathfrak{h})$ неразложима, если не существует разложения $\mathfrak{l}=\mathfrak{l}^{1} \times \mathfrak{l}^{2}$ в прямое произведение идеалов, для которого $\mathfrak{l}^{2}$ действует в $\mathfrak{n}$ тривиально и $\mathfrak{h}=\left(\mathfrak{l}^{1} \cap \mathfrak{h}\right) \times\left(\mathfrak{l}^{2} \cap \mathfrak{h}\right)$. Классификация в общем случае тривиально сводится к случаю неприводимых троек.

В отличие от случая компактного стабилизатора, ступень нильпотентности алгебры $\mathfrak{n}$ может быть больше 2. Однако классификация в случае, когда ступень нильпотентности больше 2 , несложно сводится к случаю $\mathfrak{h}=\mathfrak{l}$, который был разобран в [5]. Мы полагаем $\mathfrak{z}=[\mathfrak{n}, \mathfrak{n}]$ и обозначаем через $\mathfrak{v} L$-инвариантное дополнение к $\mathfrak{z}$ в $\mathfrak{n}$.

Мы говорим, что тройка $(\mathfrak{n}, \mathfrak{l}, \mathfrak{h})$ насыщена, если $\operatorname{dim} \mathfrak{z}(\mathfrak{l})=1, \mathfrak{z}(\mathfrak{l})$ представляется в $\mathfrak{v}$ нетривиально и $\mathfrak{n}_{\mathfrak{l}}(\mathfrak{h})=\mathfrak{h}$. Классификацию всех слабо коммутативных троек можно свести к классификации насыщенных.

Пусть $\mathfrak{l}_{0}$ - редуктивная алгебра Ли, $\mathfrak{h}_{0}$ - ее редуктивная подалгебра, а $\mathfrak{n}$ - нильпотентная алгебра Ли, в которой действует дифференцированиями алгебра Ли $\mathfrak{l}_{0} \times \mathfrak{s l}(2)$. Положим $\mathfrak{l}=\mathfrak{l}_{0} \times \mathfrak{s l}(2) \times \mathfrak{s p}(2 n), \mathfrak{h}=\mathfrak{h}_{0} \times \mathfrak{s l}(2) \times \mathfrak{s p}(2 n-2)$, где идеал $\mathfrak{s l}(2)$ алгебры $\mathfrak{h}$ вложен диагонально в $\mathfrak{s l}(2) \times \mathfrak{s p}(2 n)$. Мы говорим, что тройка $(\mathfrak{n}, \mathfrak{l}, \mathfrak{h})$ получена из тройки $\left(\mathfrak{n}, \mathfrak{l}_{0} \times \mathfrak{s l}(2), \mathfrak{h}_{0} \times \mathfrak{s l}(2)\right) \mathfrak{s l}(2)$-удвоением.

Наконец, нам понадобится одно обозначение. Для векторного пространства $V$, снабженного кососимметрической формой $\omega$, мы обозначаем через $\mathfrak{h e i s}(V, \omega)$ алгебру Ли, которая совпадает с $V \oplus \mathbb{C} z$ как векторное пространство, а $\left[v_{1}, v_{2}\right]=\omega\left(v_{1}, v_{2}\right) z$,

Работа выполнена при поддержке РФФИ (грант № 05-01-00988). 
$\left[z, v_{1}\right]=0, v_{1}, v_{2} \in V$. Когда мы пишем $\mathfrak{h e i s}(V)$, то имеем в виду, что кососимметрическая форма, участвующая в определении, невырождена.

Теорема 1. Следующие тройки насыщены, неразложимы и слабо коммутативны. Обратно, любая насыщенная неразложимая слабо-коммутативная тройка есть одна из перечисленных.

(1) $\left(\mathfrak{h e i s}\left(\mathbb{C}^{2 m+2 n}\right), \mathbb{C} \times \mathfrak{s p}(2 m+2 n), \mathbb{C} \times \mathfrak{s p}(2 m) \times \mathfrak{s p}(2 n)\right),\left(\mathfrak{h e i s}\left(\mathbb{C}^{2 n}\right), \mathbb{C} \times \mathfrak{s p}(2 n) \times\right.$ $\mathfrak{s p}(2 n), \mathbb{C} \times \mathfrak{s p}(2 n)),\left(\mathfrak{h e i s}\left(\mathbb{C}^{7} \otimes \mathbb{C}^{2}\right), \mathbb{C} \times \mathfrak{s o}(7) \times \mathfrak{s l}(2), \mathbb{C} \times G_{2} \times \mathfrak{s l}(2)\right)$.

(2) $(\mathfrak{n}, \mathfrak{l}, \mathfrak{h})$ получается однократным (соответственно двукратным $\mathfrak{s l}(2)$-удвоением из тройки вида $\left(\mathfrak{h e i s}\left(\mathbb{C}^{2 n}\right), \mathbb{C} \times \mathfrak{l}_{1}, \mathbb{C} \times \mathfrak{l}_{1}\right)$, где $\mathfrak{l}_{1} \subset \mathfrak{s p}(2 n)$ - одна из следующих линейных алгебр Ли: $\mathfrak{s p}\left(2 m_{1}\right) \otimes \mathfrak{s o}\left(m_{2}\right) \subset \mathfrak{s p}\left(2 m_{1} m_{2}\right)\left(m_{1}=1\right.$ или $\left.m_{2}=3,4\right), \mathfrak{s p i n}(2 k+1) \otimes \mathfrak{s l}(2) \subset \mathfrak{s p}\left(2^{k+1}\right), k=3,4, G_{2} \otimes \mathfrak{s l}(2) \subset \mathfrak{s p}(14)($ coomветственно $\mathfrak{s p}(2 m) \otimes \mathfrak{s o}(4) \subset \mathfrak{s p}(8 m))$.

(3) $(\mathfrak{n}, \mathfrak{l}, \mathfrak{h})$ получается однократным или двукратным $\mathfrak{s l}(2)$-удвоением троек $\left(\mathfrak{n}, \mathfrak{l}_{1}, \mathfrak{l}_{1}\right)$ из следующего списка:

(a) $\mathfrak{l}_{1}=\mathbb{C} \times \mathfrak{s l}(2 n) \times \mathfrak{s o}(m), n>1, m=3,4, \mathfrak{v}=\mathbb{C}^{2 n} \otimes \mathbb{C}^{2 m}, \mathfrak{z}=\bigwedge^{2} \mathbb{C}^{2 n}$;

(b) $\mathfrak{l}_{1}=\mathbb{C} \times \mathfrak{s l}(5) \times \mathfrak{s l}(2), \mathfrak{v}=\bigwedge^{2} \mathbb{C}^{5} \otimes \mathbb{C}^{2}, \mathfrak{z}=\left(\mathbb{C}^{5}\right)^{*}$

(c) $\mathfrak{l}_{1}=\mathbb{C} \times \mathfrak{s l}(n) \times \mathfrak{s l}(2), \mathfrak{v}=\mathbb{C}^{n} \otimes \mathbb{C}^{2}, \mathfrak{z}=S^{2} \mathbb{C}^{n}$;

(d) $\mathfrak{l}_{1}=\mathfrak{s o}(10) \times \mathfrak{s l}(2), \mathfrak{v}=\mathbb{C}^{16} \otimes \mathbb{C}^{2}, \mathfrak{z}=\mathbb{C}^{10}$ (здесъ $\mathbb{C}^{16}$ обозначает полуспи-

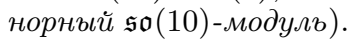

Приведем теперь некоторые соображения, использованные при классификации. Основным соображением является следующая теорема, обобщающая теорему 1 из [4].

Теорема 2. Пусть $\mathfrak{l}^{1}, \mathfrak{l}^{2}-$ произволъные дополнителъные идеалы в $\mathfrak{l}$, для которых $\mathfrak{h}=\mathfrak{h}^{1} \times \mathfrak{h}^{2}, \mathfrak{h}^{i}:=\mathfrak{l}^{i} \cap \mathfrak{h}$. Пусть $\beta, \gamma-$ элементы общего положения в $\left(\mathfrak{l}^{2} / \mathfrak{h}^{2}\right)^{*}$, $\left(\mathfrak{l}^{1} / \mathfrak{h}^{1}\right) \oplus \mathfrak{n}^{*}$ соответственно. Тройка $(\mathfrak{n}, \mathfrak{l}, \mathfrak{h})$ слабо коммутативна тогда и только тогда, когда выполнены следующие три условия:

(1) $\mathfrak{l}^{2} \subset \mathfrak{h}^{2}+\left(\mathfrak{h}^{1}+\mathfrak{l}^{2}\right)_{\gamma}$

(2) тройка $\left(\mathfrak{n}, \mathfrak{l}^{1} \times \mathfrak{h}_{\beta}^{2}, \mathfrak{h}^{1} \times \mathfrak{h}_{\beta}^{2}\right)$ слабо коммутативна;

(3) пара $\left(\left(\mathfrak{h}^{1} \times \mathfrak{l}^{2}\right)_{\gamma}, \mathfrak{h}_{\gamma}\right)$ слабо коммутативна.

Следующие два предложения являются следствиями теоремы 2. Они играют важную роль при классификации.

ПредЛОЖенИЕ 1. Пусть $\alpha$ - элемент из z общего положения. Определим форму $\widehat{\alpha}$ на $\mathfrak{v}$ посредством $\widehat{\alpha}\left(v_{1}, v_{2}\right)=\alpha\left(\left[\mathfrak{v}_{1}, \mathfrak{v}_{2}\right]\right)$. Предположим, что группа $L_{\alpha}$ редуктивна. Тогда следующие условия эквивалентны:

(1) тройка $(\mathfrak{n}, \mathfrak{l}, \mathfrak{h})$ слабо коммутативна;

(2) $\mathfrak{l}_{\alpha}+\mathfrak{h}=\mathfrak{l}$ и тройка $\left(\mathfrak{h e i s}(\mathfrak{v}, \widehat{\alpha}), \mathfrak{l}_{\alpha}, \mathfrak{h}_{\alpha}\right)$ слабо коммутативна.

ПредЛОЖенИЕ 2. Пусть $\mathfrak{l}_{0}-$ редуктивная алгебра Ли, $\mathfrak{h}_{0}-$ редуктивная подалгебра в $\mathfrak{l}_{0}, \mathfrak{n}-$ некоторая нильпотентная алгебра Ли, в которой $\mathfrak{l}_{0} \times \mathfrak{s l}(2)$ действует дифференцированиями. Тогда следующие условия эквивалентны:

(1) тройка вида $(\mathfrak{n}, \mathfrak{l}, \mathfrak{h})$, где $\mathfrak{l}=\mathfrak{l}_{0} \times \mathfrak{s l}(2) \times \mathfrak{s p}(2 n), \mathfrak{h}=\mathfrak{h}_{0} \times \mathfrak{s l}(2) \times \mathfrak{s p}(2 n-2)$, идеал $\mathfrak{s l}(2)$ вложен в $\mathfrak{s l}(2) \times \mathfrak{s p}(2 n)$ диагонально, слабо коммутативна.

(2) тройка $\left(\mathfrak{n}, \mathfrak{l}_{0} \times \mathfrak{t}(1), \mathfrak{l}_{0} \times \mathfrak{t}_{1}\right)$, где $\mathfrak{t}_{1} \subset \mathfrak{s l}(2)-$ картановская подалгебра, слабо коммутативна, $a\left(\mathfrak{h}_{0 \gamma_{1}} \times \mathfrak{s l}(2)\right)_{\gamma} \not \subset \mathfrak{h}_{0}$, где $\gamma_{1}$ - элемент общего положения 8 $\left(\mathfrak{l}_{0}, \mathfrak{h}_{0}\right)^{*}$.

\section{Список литературы}

[1] Э. Б. Винберг, УМН, 56:1 (2001), 3-62. [2] Э. Б. Винберг, Тр. ММО, 2003, №64, 54-89. [3] O. Yakimova, Gelfand pairs, Dissertation, Rheinische Friedrich-Wilhelms-Universität Bonn (Bonn, 2004), Bonner Math. Schriften, 374, Universität Bonn, Mathematisches Institut, Bonn, 2005. [4] O. Yakimova, Transform. Groups, 11:2 (2006), 305-335. [5] И. В. Лосев, Тр. ММО, 2006, № 67 , 228-255.

И. В. Лосев (I. V. Losev)

Московский государственный университет им. М. В. Ломоносова

E-mail: ivanlosev@yandex.ru
Представлено Э. Б. Винбергом Принято редколлегией 09.02.2007 